\title{
Libertad, desarrollo humano y bienestar subjetivo: una revisión crítica
}

\author{
Freedom, Human Development And Subjective Well-Being: A Critical Review
}

\author{
Rafael Domínguez Martín'
}

Copyright: ๑ 2019

Revista Internacional de Cooperación y Desarrollo.

Esta revista proporciona acceso abierto a todos sus contenidos bajo los términos de la licencia creative commons AtribuciónNoComercial-SinDerivar 4.0 Internacional (CC BY-NC-ND 4.0)

Tipo de artículo: artículo de revisión

Recibido: septiembre de 2019

Revisado: octubre de 2019

Aceptado: octubre de 2019

Autor

1 Catedrático de Historia e Instituciones Económicas, Departamento de Economía de la Universidad de Cantabria (España). Director del Grupo de Investigación sobre Desarrollo Humano y Cooperación Internacional (Cátedra COIBA)

Contacto: rafael.dominguez@unican.es

ORCID 0000-0002-5938-0023

Cómo citar:

Domínguez Martín, R. (2019). Libertad, desarrollo humano y bienestar subjetivo: una revisión crítica. Revista Internacional de Cooperación y Desarrollo. 6(2). 50-67

DOI $10.21500 / 23825014.4548$

\section{Resumen}

El objetivo de este trabajo es revisar críticamente el papel de la libertad como determinante del bienestar subjetivo a lo largo del proceso de desarrollo humano, entendido en el sentido sociológico de la teoría postmaterialista de la modernización. Aunque la legitimidad de utilizar información subjetiva a partir de encuestas para medir el desarrollo humano ha sido cuestionada por el problema de las preferencias adaptativas, rechazarla por principio para explicar el bienestar resulta contradictorio con la premisa fundante del paradigma del desarrollo humano, dada su defensa irreductible de la agencia individual.

La hipótesis de partida es que la literatura de los estudios del desarrollo que busca explicar la variación del bienestar subjetivo en el proceso de desarrollo humano tiende a reducir la libertad de elección, como libertad negativa (no interferencia), a la libertad económica del consumidor, lo que conduce a falsas pistas y sesgos eurocéntricos.

La conclusión de la revisión es que resulta necesario recuperar el concepto integral de libertad, como libertad positiva o autorrealización, asociado a la tradición aristotélica-marxista-polanyiana, que enlaza con los planteamientos originales de Amartya Sen.

Palabras clave: libertad; desarrollo humano; bienestar subjetivo; teoría de la modernización.

\section{Abstract}

The objective of this paper is to review critically the role of freedom as a determinant of subjective well-being throughout the process of human development, understood in the sociological sense of the post-materialist theory of modernization. Although the legitimacy of using subjective information from surveys to measure human development has been questioned by the problem of adaptive preferences, rejecting it in principle to explain well-being is contradictory with the foundational premise of the human development paradigm, given his irreducible defense of individual agency.

The starting hypothesis is that the literature of development studies that seeks to explain the variation of subjective well-being in the human development process tends to reduce freedom of choice, such as negative freedom (non-interference), to consumer economic freedom, which leads to false tracks and Eurocentric biases.

The conclusion of the review is that it is necessary to recover the integral concept of freedom, as positive freedom or self-realization, associated with the Aristotelian-Marxist-Polanyian tradition, which links to the original approach of Amartya Sen.

Keywords: freedom; human development; subjective well-being; modernization theory. 


\section{Introducción}

El objetivo de este trabajo es revisar críticamente el papel de la libertad como determinante del bienestar subjetivo a lo largo del proceso de desarrollo humano, entendido en el sentido sociológico que le da la teoría postmaterialista de la modernización (Welzel 2011). La legitimidad de utilizar la información subjetiva a partir de encuestas (tanto en términos evaluativos o de satisfacción vital, como hedónico o de experimentación de felicidad) para medir el desarrollo humano ha sido cuestionada por el problema de las expectativas o preferencias adaptativas (Stewart 2014; Burns 2016). Pero rechazar por principio esta información subjetiva para explicar el bienestar resulta contradictorio con la premisa fundante del paradigma del desarrollo como ampliación de las capacidades de las personas o desarrollo humano (Sen, 1999), dada su defensa irreductible de la agencia individual en tanto que capacidad autónoma de realizar juicios morales y de actuar (Croker \& Robeyns, 2010; Dang, 2014; Hirai, Comin \& Ikemoto, 2016), autonomía que es ampliamente reconocida como una necesidad psicológica innata (Ryan \& Deci, 2000); además de que la utilización de las evaluaciones subjetivas arrojan resultados promisorios que complementan otros datos objetivos para medir y explicar el bienestar (Kingdon \& Kinght, 2006; Dang, 2014).

Justificada la relevancia y pertinencia del planteamiento del problema, su abordaje se realiza desde la sociología histórica y la economía política en la tradición del realismo crítico (Lawson, 1997; Steinmetz, 1998). La hipótesis de partida es que la literatura de los estudios del desarrollo que busca explicar la variación del bienestar subjetivo en el proceso de desarrollo humano tiende a reducir la libertad de elección, como libertad negativa (no interferencia), a la libertad económica del consumidor, lo que conduce a falsas pistas y sesgos eu- rocéntricos. La conclusión de la revisión es que resulta necesario recuperar el concepto integral de libertad, como libertad positiva o autorrealización, asociado a la tradición aristotélica-marxista-polanyiana, que enlaza con los planteamientos originales de Amartya Sen.

El trabajo se divide en cinco secciones. En la primera se discute el concepto trivalente de libertad como no interferencia (libertad negativa), self-realization (libertad positiva) y no dominación. En la segunda se examinan las ambivalencias y complejidades del pensamiento de Sen en sus tres trabajos canónicos sobre el desarrollo como ampliación de las libertades o desarrollo humano (Sen, 1985; 1999; 2009). En la tercera se analizan los hallazgos principales de la investigación reciente de los estudios del desarrollo sobre la relación entre libertades y bienestar subjetivo, considerando el elemento de la religiosidad como un factor complementario, más que sustitutivo, de la libertad. En la cuarta se cuestiona la preferencia dada a la libertad como no interferencia en dicha literatura, en la medida en que se reduce la libertad de elección a libertad económica del consumidor.

El trabajo cierra con unas consideraciones finales que reivindican una vuelta al concepto de libertad positiva de Marx, Polanyi y la teoría crítica como factor explicativo del bienestar subjetivo y sus variaciones entre personas y países, lo que cuestiona el sesgo eurocéntrico de la teoría postmaterialista de la modernización.

\section{Tres conceptos de libertad}

El concepto de libertad que se maneja en el campo del bienestar subjetivo y los estudios sobre la felicidad remite al paradigma de las capacidades como libertades de Sen, o más bien a una interpretación sesgada del mismo hacia las libertades negativas en el contexto de la revisión de la teoría de la modernización (Welzel, 2011). De ahí la nece- 
sidad de fijar las definiciones que desde la filosofía moral y política se han desplegado a partir de mediados del siglo xx sobre los principales conceptos de libertad: libertad negativa, libertad positiva y libertad como no dominación.

En un ciclo de conferencias radiofónicas sobre los enemigos de la libertad recogidas cinco décadas después en un libro, Isaiah Berlin afirmó en 1952 que la "esencia de la libertad reside siempre en la capacidad de elegir lo que deseas elegir, porque deseas así eliges" (Berlin, 2002, p.111). Berlin aclara que esa definición deriva de un planteamiento moral sobre la responsabilidad, que es la piedra angular del radical anti-determinismo que caracteriza a su pensamiento: la capacidad de elegir "presupone la noción de responsabilidad" (Berlin, 1998, p.324).' La noción de responsabilidad es central en el debate sobre el libre albedrío, o "libertad de la voluntad", la "libertad en el sentido metafísico" como dijo Max Horkheimer (1962, p.11), debate que, desde el precedente de los estoicos (aunque la fuente original es Aristóteles), recorre la historia del cristianismo, con las figuras destacadas de Agustín de Hipona y Tomás de Aquino: los seres humanos no están determinados en sus acciones, sino que, como seres racionales, tienen la capacidad de elegir, lo que implica que deben asumir (son responsables de) las consecuencias de sus decisiones.

A partir de esta explicitación ex post de la definición esencial o constitutiva de la libertad como derivada de la noción de responsabilidad y su conexión con la libertad metafísica o libertad de la voluntad (el libre albedrío), se entiende mejor la famosa distinción de Berlin entre libertad negativa y libertad positiva, que data de 1958: la libertad negativa se refiere a la "no interfe-

1"la responsabilidad implica la capacidad de elegir entre blanco y negro, correcto e incorrecto, placery deber; $y$, en un sentido más amplio, entre formas de vida, formas de gobierno, y todas las constelaciones de valores morales" (Berlin, 1969, p.324). rencia", a la freedom to o libertad de (elegir, hacer, pensar, expresarse), en la tradición de Hobbes, Locke, Montesquieu, Bentham, Tocqueville y Mill; y la libertad positiva que es la "autorrealización" (self-realization), la freedom for o libertad para de la tradición de Herder, Rousseau, Fichte, Hegel y Marx (aunque también de Aristóteles y Mill, como luego recordará Sen). Para Berlin, ambas libertades acaban chocando, y aunque en principio parece no haber un juicio favorable de una sobre otra ${ }^{2}$ la conclusión final es que la libertad negativa ofrece "un ideal más verdadero y más humano que los fines que aquellos que buscan en las grandes estructuras disciplinarias y autoritarias el ideal de autocontrol «positivo» para las clases, los pueblos o la humanidad entera" (Berlin, 1969, p.216).

Sin embargo, hay un pecado original de olvido en el ensayo de Berlin, porque la distinción entre libertad negativa y positiva, así como la literalidad de su terminología, proceden de la tradición marxista y, en concreto, del libro de Erich Fromm El miedo a la libertad que se publicó en 1941 y que Berlin no cita hasta 1969. La razón de esta omisión, al margen del contexto del condicionante de la Guerra Fría (Skinner, 2002) es doble: para Fromm la esencia de la libertad es la libertad negativa, pero en un sentido activo (liberación), no pasivo (ausencia de interferencia) que es como luego la presentará Berlin; y, además, según Fromm esa libertad negativa es un medio para alcanzar el telos de la libertad positiva, libertad de aroma determinista (porque está en la esencia del ser humano) que para la tradición liberal de la filosofía moral y política fundada por Berlin es solo un sucedáneo de la libertad.

Fromm (1941, p.30) arranca con la siguiente cuestión: “¿es la libertad solamente

2"el énfasis en la libertad negativa, como regla, deja más caminos para que los transiten individuos o grupos; la libertad positiva, como regla, abre menos caminos, pero con mejores razones o mayores recursos para moverse a lo largo de ellos" (Berlin, 1969, p.49). 
ausencia de presión exterior o es también presencia de algo? Y, siendo así, ¿Qué es ese algo?" Su respuesta es que "la existencia humana y la libertad son inseparables desde un principio. La noción de libertad se emplea aquí no en el sentido positivo de «libertad para», sino en el sentido negativo de «libertad de», es decir, liberación de la determinación instintiva del obrar" (Fromm, 1941, p.58). Esa liberación está narrada en el mito del Paraíso, que describe "el primer acto de libertad" propiamente humano (racional) como "un acto de elección" que es "un acto de desobediencia” (Fromm, 1941, pp.59-60). Pero "liberarse de" ("nuestra definición de la libertad como rechazo de la sumisión a todo poder superior") no es lo mismo que "liberarse para" (alcanzar "la realización plena de las potencialidades del individuo, así como con su capacidad para vivir activa y espontáneamente"), aunque las dos libertades (negativa y positiva) están conectadas por la idea de liberación, ya que la segunda se basa en "el principio de que no existe poder superior al del yo individual" o, dicho de otro modo, "que el desarrollo y la realización de la individualidad constituyen un fin que no puede ser nunca subordinado a propósitos a los que se atribuyen una dignidad mayor" (Fromm, 1941, pp.60, 302, 305, 308). En este marco, Fromm plantea la tesis de que la libertad de elección no ha permitido al ser humano alcanzar "la libertad en el sentido positivo de la realización de su ser individual, esto es, la expresión de su potencialidad intelectual, emocional y sensitiva" (Fromm, 1941, p.24), más bien lo que se ha producido con el desarrollo humano (una afirmación del individuo frente a las autoridades tradicionales) es un aumento de la brecha entre libertad de y libertad para, de modo que "la libertad -como libertad negativa- conduce hacia nuevas cadenas" (Fromm, 1941, pp.63, 291).

Este planteamiento, por el cual la libertad negativa (la liberación de todo poder superior al ser humano) es el medio para el fin de la libertad positiva o self-realization y como tal medio ha fracasado, era anatema para los liberales y remite al debate soterrado entre Friedrich Hayek y Karl Polanyi, contemporáneo del trabajo de Fromm. Hayek critica tempranamente el ideal socialista de libertad que incluye los derechos económicos y sociales además de los civiles y políticos (Hayek, 1938, pp.666, 670), y en la bibliografía de su best-seller cita un trabajo de Polanyi (The Contempt of Freedom, 1940) como representativo de esa noción de libertad de la necesidad que el austríaco considera tan solo una forma de poder (ya que se alcanza por medio de la planificación estatal) y es una amenaza a la (genuina) libertad individual de elección y la prosperidad ( $\mathrm{Ha}$ yek, 1944, p.27). Polanyi responde a Hayek contraponiendo a esta libertad de elección, la "libertad de la necesidad" (Polanyi, 1944, p.171) y, sin citarlo, arremete contra la tesis liberal de que la planificación y la intervención estatal son "la negación de la libertad": por el contrario, sus objetivos de justicia y bienestar pueden "suponer el comienzo de una era de libertades sin precedentes, de lograr la libertad, no sólo para unos pocos sino para todos" (Polanyi, 1944, p.265).

Tras este debate, Berlin propone una síntesis social-liberal para lo cual invierte la primera parte del argumento de Fromm: la libertad negativa (reducida a una visión pasiva de no interferencia) es la esencia de la libertad, la "libertad como tal", mientras que las "condiciones suficientes para su activo ejercicio" son las que definen la libertad positiva (la libertad de la necesidad de Polanyi), que "incluye una amalgama de otras cosas deseables -igualdad, justicia, felicidad, conocimiento, amor, creatividad y otros fines que los hombres buscan por su propio beneficio" (Berlin, 1969, p.50). Así todo, al concluir la revisión de sus ideas sobre la libertad después de cuarenta años, Berlin (1998) concede que ambos sentidos 
de la libertad se han pervertido con el paso del tiempo: la libertad negativa porque ha sido reducida a libertad económica; $y$ la libertad positiva porque se ha invertido transformándose en obediencia a una autoridad que decide cuál es el verdadero interés de los individuos sin consultarlos. Aunque Berlin da prioridad a su concepto de libertad negativa-y por ello critica como "un error teórico" y político en el énfasis en la libertad positiva (Berlin, 1969, p.50)-, la complementariedad de los dos sentidos es obvia en su pensamiento: como señala Skinner (2002, p.241), se trata de "estar libre de interferencias para hacer algo". Al comentar que la libertad "en su sentido político" es "colindante con la ausencia de intimidación o dominación" (Berlin, 1969, p.48), Berlin anticipa, además, "el tercer concepto de libertad", una tradición que conceptualiza la idea de libertad negativa "no como ausencia de interferencia, sino como ausencia de dependencia" de poderes arbitrarios (Skinner, 2002, p.255), libertad que es "la antinomia de la dominación", como dirá el principal exponente de esta corriente, el politólogo y filósofo moral Philip Pettit (1997, p.273).

Los teóricos de la libertad como no dominación consideran que el concepto de libertad negativa (no interferencia) es demasiado estrecho (Kramer, 2011) y políticamente buscan estirar los límites del liberalismo con una propuesta de republicanismo democrático o neo-republicanismo, en la tradición de Polibio, Cicerón, Tito Livio, Maquiavelo, y que tiene como referentes a los radicales ingleses del siglo XVII, Rousseau, los revolucionarios franceses de 1789 , Tom Paine, Mary Wollstonecraft, John Stuart Mill o Harriet Taylor (Pettit, 1997, p.2016). En su diálogo con Sen, Pettit considera que el enfoque de las capacidades está muy sesgado hacia la libertad individual de oportunidades que, por la complejidad para su operacionalización, tiende a ser reducida a la libertad de elección de los neoliberales y no toma en cuenta el aspecto de proceso de la libertad en forma de acción colectiva (Pettit, 2010). Como alternativa a estas insuficiencias, Pettit propone en clave neo-republicana una libertad social centrada en el estatus, donde se valora cuán libre es un ciudadano. Este concepto clásico de ciudadanía asociado a la libertad como no dominación implica el disfrute de derechos en términos de libertad de elección y de igual distribución o acceso a esos derechos, que el Estado debe garantizar frente a otros poderes públicos o privados (grupos de presión, empresas, patriarcas familiares) de carácter arbitrario: "la libertad entendida como no dominación es una propiedad que solo puede materializarse si se asegura la igualdad de su disfrute", por tanto, la igualdad es una condición de la libertad (Pettit, 2009, p.59).

Para el republicanismo democrático, los derechos igualmente distribuidos, entendidos como libertades básicas o protecciones de un mismo rango de elección, deben satisfacer constitutivamente el requisito mínimo de la dignidad, (por cierto, el mismo que toma Sen de Adam Smith): que el individuo pueda vivir o presentarse ante los demás sin vergüenza, discriminación o humillación porque goza de un igual estatus objetivo y subjetivo de no dominado. ${ }^{3}$ En tal supuesto, lo que se necesita son arreglos institucionales de modo que la democracia sirva a la causa de la libertad (Pettit, 2010; 2012; 2016). Esto significa un "control controlado": el Estado tiene el deber de "regular a los agentes poderosos y agencias que pudieran ejercer un cierto control sobre los ciudadanos ordinarios" (Pettit, 2009, p.50), pero, a la vez, el Estado está sujeto al control político democrático de las personas y así

${ }^{3}$ En este punto, tanto el enfoque de Axel Honneth (2006) de lucha por el reconocimiento, como los conceptos de desposesión forzada y desposesión constitutiva de Judith Butler (Cano, 2017) encajan en el marco teórico del republicanismo enriqueciéndolo y pueden servir de guía normativa para evaluaciones complementarias del concepto de libertad como no dominación (Pereira, 2008). 
"la agencia que nos protege de la dominación por otros no perpetrará la dominación en el acto de hacerlo" (Pettit, 2010, p.112). Se trata de "asegurar que las personas comparten igualmente el control de la ley, dando al demos o pueblo el kratos o poder", lo que desborda la concepción liberal de la democracia representativa para situarse en la democracia participativa y contestataria (Pettit, 2016, p.17).

El tercer concepto de libertad, en definitiva, confronta la ortodoxia de la libertad neoliberal de elección que busca un Estado mínimo con un mercado máximo, que se concreta en la libertad como no dominación solo para unos pocos, esto es, la libertad de contratos entre privados sin tener en cuenta el diferente nivel de empoderamiento de las partes, que ya en su día denunció Marx al criticar la libertad como no interferencia de Bentham (Pettit, 1997, p.2016). Por esto, aunque el tercer concepto de libertad como no dominación sea considerado dentro de una idea más amplia de la libertad negativa y rechace explícitamente ser parte del concepto de libertad positiva (Pettit, 1997), el neo-republicanismo acepta y promueve que las libertades positivas (los derechos económicos y sociales) sean habilitantes de la no dominación (Pettit, 2016), lo que ha dado lugar a propuestas políticas, que, reclamándose de este tercer concepto de libertad, ya son abiertamente socialistas (Casassas, 2008, 2018).

Antes de analizar lo que Sen entiende por desarrollo como libertad (como proceso de ampliación de las libertades concretadas en forma de derechos), es necesario constatar a estas alturas de la exposición que en el debate de la filosofía moral y política sobre los conceptos de libertad parece existir un doble consenso conceptual y axiológico de sesgo liberal que es la doxa delimitante de la discusión: en clave conceptual, hay consenso sobre la necesidad de identificar la libertad negativa con la ausencia de impedimentos y la presencia de oportunidades, mientras que la libertad positiva se relaciona con el aspecto de los procesos y los cumplimientos (Kukathas, 2007; Kramer, 2011); en clave axiológica hay consenso en torno a que la esencia de la libertad es esa capacidad de elegir de la que habló Berlin, mientras que los sentidos de la libertad positiva son "superfluos", ya que existe una miríada de términos sustitutivos (Kramer, 2011, p.1451). ${ }^{4}$

\section{Sen y la pluralidad de las libertades}

Sen comparte en general ese doble consenso (Cuadro 1), aunque, dada la ambivalencia de su pensamiento (quizá por el apego al justo medio aristotélico o al espectador parcial smithiano, quizá por el afán de alcanzar una síntesis que pudiera permanecer dentro de la economía mainstream), se necesita desentrañar la complejidad y valorar los matices de lo que propone. Y lo que propone se puede resumir en una variación -de ecos berlinianos-sobre la libertad positiva como condición habilitante para el ejercicio de la libertad negativa, definida como libertad de elección (el aspecto de las oportunidades y la agencia individual), pero de un modo tan amplio (elegir cómo vivir) que admite una lectura alternativa atenta al aspecto de los procesos y la acción colectiva. Por tanto, Sen no es un defensor de la equiparación conceptual y normativa de la libertad como libertad económica del consumidor, sino que su planteamiento es mucho más abierto y pluralista (Dang, 2014), especialmente cuando expresa sus puntos de vista personales en entrevistas, como en la que defiende su idea de libertad, equiparable a la libertad positiva en la tradición aristotélico-marxista. ${ }^{5}$

${ }^{4}$ Este autor lista los siguientes términos del "credo de la libertad positiva": self-fulfillment, self-realization, self-expression, self-mastery, autonomy, self-reliance, self-control, self-determination, flourishing, self-development, self-direction, popular political participation y active citizenship.

5 "si de lo que se trata es de definir un fin social, me parece que podríamos hablar de la libertad positiva, la libertad de hacer y ser aquello que es posible en la rea- 
Cuadro 1.

El consenso liberal sobre los tres conceptos de libertad

\begin{tabular}{|c|c|c|}
\hline Concepto de libertad & Contenido axiológico y funcional & Derechos a que da lugar \\
\hline $\begin{array}{l}\text { libertad negativa } \\
\text { agency freedom }\end{array}$ & $\begin{array}{c}\text { libertad de } \\
\text { no interferencia } \\
\text { oportunidades } \\
\text { capacidad de elegir }\end{array}$ & $\begin{array}{l}\text { Derechos de primera generación } \\
\text { (civiles y políticos) }\end{array}$ \\
\hline $\begin{array}{l}\text { libertad positiva } \\
\text { well-being freedom }\end{array}$ & $\begin{array}{l}\text { libertad para } \\
\text { self-realization } \\
\text { procesos } \\
\text { cumplimientos }\end{array}$ & $\begin{array}{l}\text { Derechos de segunda generación } \\
\text { (económicos, sociales y culturales) }\end{array}$ \\
\hline libertad como no dominación & $\begin{array}{l}\text { estar libre de } \\
\text { ausencia de dependencia } \\
\text { reconocimiento } \\
\text { acción colectiva }\end{array}$ & $\begin{array}{l}\text { Derechos de primera y de segunda } \\
\text { generación }\end{array}$ \\
\hline
\end{tabular}

En su trabajo seminal sobre el tema, Sen distingue el "aspecto del bienestar" ("libertad para alcanzar el bienestar" o well-being freedom) y el "aspecto de la agencia" ("un concepto más amplio de libertad", referido a lo que "una persona es libre de hacer y lograr en la persecución de cualesquiera fines o valores que él o ella considere importantes" o agency freedom) (Sen, 1985, p.169, 201, 203). Este concepto más amplio luego será resumido en su famosa definición del desarrollo como "la expansión de la libertad humana para vivir el tipo de vidas que las personas tienen razones para valorar" (Sen, 1999, p.295). Pues bien, "la expansión de la libertad" es "tanto el fin primario como el medio principal del desarrollo" (Sen, 1999, p.xii), o, dicho de otra manera, hay un "«papel constructivo» y un «papel instrumental» de la libertad en el desarrollo" (Sen, 1999, p.36). Conviene aclarar que el punto de partida de Sen es normativo y queda circunscrito al individualismo ético de Kant o Rawls

lidad, no sólo la libertad respecto de las interferencias de los otros. La libertad de hacer y ser lo que uno puede considerar oportuno, aquello que tiene razones para estimar oportuno. Esta capacidad racional de los seres humanos de elegir sus propios fines, de perseguirlos en libertad, me parece que es el gran hilo conductor de un discurso que parte de la democracia social de Aristóteles para llegar a los pensadores socialistas europeos, incluidos los escritos de Marx" (Sen, 1993, p.79).
(Otano, 2015), ya que trata "las libertades de los individuos como los building blocks" de ese paradigma de las capacidades en construcción (Sen, 1999, p.18). Esto le lleva a dar prioridad en el análisis al "aspecto de las oportunidades" para lograr resultados valiosos para los individuos frente al "aspecto de los procesos" que implican la construcción de mecanismos colectivos para la toma de decisiones (Sen, 1999, pp.17, 291). El interés de Sen es doble: estudiar el desarrollo "como un proceso de ampliación de las libertades sustantivas que se conectan unas con otras" (Sen, 1999, p.8) o dimensión constitutiva; ${ }^{6}$ e integrar las dos nociones de Berlin (Sen también olvida a Fromm) a través de diversas clasificaciones de "derechos, oportunidades y títulos (entitlements), que contribuyen a la expansión de la libertad humana en general y así promueven el desarrollo" (Sen, 1999, p.37) o dimensión instrumental de las libertades.

Desde esta perspectiva analítica e instrumental, Sen distingue cinco tipos de libertades que incluyen las dos dimensiones (positiva y negativa) de Berlin y que se so-

\footnotetext{
${ }^{6}$ El concepto de libertad sustantiva en Sen se refiere a aquellas libertades como "la libertad de participación política o la oportunidad de recibir educación básica o atención médica", que "se encuentran entre los componentes constituyentes del desarrollo" (Sen, 1999, p.5).
} 
lapan parcialmente con la distinción entre "procesos que permiten la libertad de acciones y decisiones y oportunidades que la gente tiene, dadas sus circunstancias personales y reales" (Sen, 1999, p.17). En este punto Sen (1999, p.38) salta directamente a la terminología (no a la tesis) de las "libertades instrumentales" como libertades concretas que suponen derechos de distinta naturaleza. Se trata de: 1) libertades políticas, 2) facilidades económicas, 3) oportunidades sociales, 4) garantías de transparencia y 5) seguridad protectora. Claramente 3 y 5 corresponden a las libertades positivas o derechos de segunda generación (económicos y sociales), mientras 1,2 y 4 son libertades negativas o derechos de primera generación (civiles y políticos).

Las libertades políticas "son elementos constitutivos de la libertad del hombre", que resultan "directamente importantes por sí mismos, y no pueden justificarse indirectamente en términos de sus efectos sobre la economía" e "incluyen los derechos políticos asociados con las democracias en un sentido amplio" (Sen, 1999, pp.16-17, 38). Estas libertades políticas o "derechos políticos liberales" adquieren en el esquema de Sen "una preeminencia general" (Sen, 1999, p.148) por tres razones que se categorizan respectivamente como directa (constitutiva), instrumental y constructiva de preferencias.

Las facilidades económicas, sin embargo, no son derechos exigibles en el mismo sentido que las libertades políticas: se trata de "oportunidades que los individuos disfrutan o recursos económicos que utilizan para propósitos de consumo, producción o intercambio"; y estos títulos (entitlements), que dependen de los recursos propios o disponibles (cuya distribución se considera dada) y condiciones de intercambio ("precios relativos y funcionamiento de los mercados"), se amplían con el crecimiento económico (Sen 1999, p.38). Aunque Sen re- conoce que "las cuestiones distributivas son importantes" (Sen 1999, p.39) esta elipsis por la que se pasa de la distribución dada de los entitlments a la ampliación de los mismos (crecimiento) por medio del mercado, señaliza los límites ortodoxos de su propuesta. Análogamente, la prevención de la corrupción, que está vinculada al papel instrumental de las garantías de transparencia, es el verdadero complemento de la libertad de empresa en una economía social de mercado, a la que de forma indirecta se refiere Sen con el concepto de facilidades económicas.

En cambio, las oportunidades sociales sí pertenecen ya al dominio de las libertades positivas: son "los arreglos que hace la sociedad para la educación, la atención médica, etc., que influyen en la libertad sustantiva para vivir mejor", y que "son importantes, no solo para la conducta de vidas privadas (como vivir una vida sana y evitar la morbilidad prevenible y la mortalidad prematura), sino también para una participación más efectiva en las actividades económicas y políticas" (Sen, 1999, p.39). Esto es aplicable asimismo al concepto de seguridad protectora, cuya función es "proporcionar una red de seguridad social para evitar que la población afectada sea reducida a la abyecta miseria, y, en algunos casos, incluso a la inanición y la muerte" (Sen, 1999, p.40). Con ello Sen se hace eco de la idea de Berlin de la libertad positiva como condición necesaria para el ejercicio de las libertades negativas. ${ }^{7}$

En resumen, aunque Sen intenta superar la dicotomía entre libertad negativa y positiva, le otorga un papel decisivo a la primera en la operacionalización de su propuesta política a través de la reivindicación de las libertades civiles y políticas adop-

\footnotetext{
7 "Los factores sociales y económicos como la educación básica, la atención primaria de salud y el seguro de empleo no solo son importantes por sí mismos, sino también por el papel que pueden desempeñar en dar las personas la oportunidad de acercarse al mundo con coraje y libertad" (Sen, 1999, p.63).
} 
tando la terminología (no la tesis) de las libertades específicas y sus traducciones en derechos concretos. En su última gran obra, Sen trata de completar este paradigma ampliando algunos puntos apenas esbozados en otros trabajos anteriores y, además, busca dar cabida al tercer concepto de libertad. Para ello introduce la distinción entre "resultado de culminación" (culmination outcome) y "resultado integral" (comprehensive outcome): el primer resultado es lo que una persona alcanza realmente gracias a la oportunidad, que Sen considera una visón "particularmente estrecha" de la oportunidad; el segundo resultado es integral y supone un enfoque más amplio porque, además de la oportunidad, toma en cuenta el proceso (Sen, 2009, p.230). Este es, en definitiva, el concepto operativo de libertad como capacidad que Sen defiende para fines evaluativos. Pero el autor se autolimita al liberalismo político, por mucha gimnasia verbal que haga: su noción de capacidad "está orientada hacia la libertad y las oportunidades, esto es, la habilidad efectiva de las personas para elegir entre vivir diferentes tipos de vidas a su alcance, en lugar de confinar su atención tan sólo a lo que puede ser descrito como la culminación -o secuela-de la elección"; dicho de otro modo, "la cuestión central es la libertad para elegir como vivir" (Sen, 2009, pp.237-238), lo que repite con otras palabras la idea de que las libertades positivas (libertad para, en este caso, elegir como vivir) son habilitantes de las negativas (libertad de elección), de ahí que reclame la inclusión de los derechos económicos y sociales (derechos de segunda generación) en el espacio de la libertad.

En coherencia con ello, Sen se defiende de las acusaciones de individualismo metodológico al reconocer que las acciones de los individuos son influidas por el entorno social que los rodea y por las múltiples adscripciones de las personas en las sociedades modernas. $Y$ en el contexto de esta discusión, es donde da cabida a la libertad como no dominación: el "poder efectivo" ("el poder de alcanzar los resultados elegidos") a través del "control sobre el proceso de elección" (Sen, 1985, pp.208-209) que caracteriza el planteamiento del republicanismo, "confiere a la libertad sustantiva una fuerza que está ausente cuando esa libertad de hacer... está condicionada a la ayuda o la tolerancia de otros" (Sen, 2009, pp.306). En todo caso, el tercer concepto de libertad para Sen es una manifestación de las características plurales de la libertad, pero la libertad como no dominación es un enfoque monotemático que no sirve para reemplazar el paradigma irreductiblemente plural de las capacidades y las libertades.

\section{Libertad y bienestar subjetivo en los estudios del desarrollo}

En la literatura de los estudios del desarroIlo la libertad, en sus concepciones negativas de no interferencia y no dominación, se ha considerado como predictor del bienestar subjetivo y determinante de la felicidad con resultados positivos y robustos (Inglehart et al., 2008; Verme, 2009; Helliwell, Huang \& Wang, 2017). Además, los arreglos institucionales habilitantes del concepto negativo de libertad ampliada (la democracia participativa con control ciudadano) han mostrado estar sistemáticamente asociados a mayores niveles de bienestar subjetivo (Frey \& Stultzer, 2000).

En la revisión crítica de la teoría funcionalista de la modernización ${ }^{8}$ a través de la lente del paradigma del desarrollo humano (Welzel, 2011) la teoría postmaterialista también otorga a la libertad un valor explicativo fundamental del bienestar subjetivo: la modernización es una transición desde la

${ }^{8}$ La modernización es un vasto conjunto de transformaciones que incluyen la creación de una economía urbana y de mercado, la diferenciación de esferas sociales, la burocratización y racionalización institucional basada en el Estado, y la secularización o declive de la religión (Ingleharty Welzel, 2005; 2007; Dobbelaere, 2007; Volpe, 2010; Bjerke, 2012; Fokas, 2012). 
sociedad de la escasez (donde el crecimiento económico y la función atenuadora de incertidumbre que tiene la religión son componentes fundamentales del bienestar) hacia la sociedad de la seguridad existencial (donde la libertad, en términos de self-expression, libertad de elección y control, toma el relevo a la religión como componente fundamental del bienestar) (Inglehart etal., 2008; Verme, 2009; Knutsen, 2011; Norris \& Inglehart, 2011; Müller, De Graaf \& Schmidt, 2014). Esto permite clasificar a los países a lo largo de la curva que describe la paradoja de Easterlin (la relación exponencial decreciente entre PIB per cápita y bienestar) en la que los 13 países latinoamericanos incluidos en el análisis aparecen como claros outliers para el período 1981-2007 (Inglehart et al., 2008): una de las dos principales razones que se ofrece para explicar lo que Rojas (2016) y Beytía (2016) consideran la paradoja latinoamericana (el disfrute de un nivel más elevado de bienestar subjetivo del que corresponde al nivel de desarrollo medido por el PIB per cápita) es la persistencia de la creencia en Dios, o más concretamente, el alto nivel de religiosidad bajo condiciones de inseguridad económica; y la otra es la relativamente elevada libertad de elección asociada a los procesos de democratización que vivió la región desde la década de 1980. Los últimos datos disponibles para 20152016 confirman la existencia de esa paradoja latinoamericana del bienestar subjetivo (Helliwell, Huang \& Wang, 2017).

Estos resultados matizan el principio general propuesto por Inglehart et al. (2008) con respecto al concepto de "liberalización social" o "tolerancia a los outgroups" (mujeres, gays y lesbianas, minorías religiosas, extranjeros, o sujetos con estilos de vida alternativos): a mayores niveles de ingreso per cápita de los países mayor es la contribución al bienestar de este factor que remite (implícitamente) al concepto de libertad como no dominación y su concepción más avanzada de la democracia (democracia participativa) y, por tanto, de la cultura política del mismo nombre (Cartocci, 2011). En todo caso, los autores se centran en la concepción más tradicional de la libertad como no interferencia, o "libertad de elección y control" (que incluye self-expression), libertad que estiman tiene el "impacto principal" sobre el incremento de la felicidad como variable intermedia dependiente de las variables independientes-aunque correlacionadas entre sí-del crecimiento económico, la democratización y el aumento de la tolerancia social (Inglehart et al., 2008, pp.270271).

El mismo trasfondo empírico de personas y países es el que usa Verme (2009) en el marco del giro psicológico que han tomado una gran parte de los estudios sobre el bienestar en los últimos años. Pero en vez de partir de las teorías de la estructura básica de la personalidad que dieron lugar a la teoría de la cultura política en que se basa la teoría postmaterialista de la modernización (Cartocci, 2011), Verme acude a las teorías conductistas de la personalidad que clasifican a las personas de acuerdo al lugar del control en el binomio internals/externals: las personas internals creen que el resultado de sus acciones depende de factores internos a ellas tales como el esfuerzo o las habilidades (el lugar de control de elección es interno a las personas) y tienen una mayor aprecio a la libertad de elección que las externals; las externals son aquellas personas que creen que los resultados de sus acciones dependen de factores externos como la suerte o el destino (el lugar de control de elección es externo a las personas). La hipótesis del trabajo es que el lugar de control de la elección actúa como un regulador del valor intrínseco que las personas otorgan a la libertad de elección (lógicamente, los internals otorgarán un valor superior a la libertad de elección que los externals y la curva de posibilidades de producción de la 
felicidad estará más arriba y hacia la derecha para los primeros). Tal hipótesis sirve para modificar la teoría del ciclo vital de las personas y de la transición hacia la sociedad postmaterial de los países. Para bajos niveles de ingreso, el crecimiento económico expande la libertad de elección y la felicidad de los individuos independientemente de su personalidad, pero a partir de un determinado umbral de ingreso y felicidad, más ingreso y más libertad pueden traducirse en más felicidad solo para los internals. Y cuando los países se mueven de la autocracia a la democracia los gobiernos tienden a favorecer el aumento de la felicidad de los internals sobre los externals; lo contrario sucede si la democracia da paso a la autocracia, apunte que deja en suspenso la hipótesis de Inglehart del avance lineal hacia la democratización con el desarrollo. Combinando la libertad de elección con los dos tipos de personalidad a partir del lugar de control, los resultados que se obtienen del análisis (260.000 individuos de 84 países en un período de 25 años) muestran que los internals tienen una mayor apreciación de la libertad de elección que los externals y que esta variable de control es necesaria para explicar la conexión entre libertad y felicidad.

Gundlach \& Opfinger (2013) permiten completar este análisis con la consideración del factor religiosidad. Los autores delimitan las relaciones contradictorias, a modo de hechos estilizados, entre bienestar subjetivo y desarrollo humano: la correlación positiva entre felicidad e ingreso (uno de los tres componentes del desarrollo humano operacionalizado en el índice del mismo nombre), la correlación positiva entre felicidad y religiosidad, y la correlación negativa entre religiosidad e ingreso. El planteamiento queda resumido en una exposición diagramática en donde las curvas de indiferencia de la felicidad (como resultantes de dos componentes: religiosidad y otros elementos) indican que un determinado nivel de felicidad se puede mantener sustituyendo el componente de felicidad por otro elemento (a mi juicio, la libertad o el ingreso), de manera que un aumento de la libertad o del ingreso pueden causar un descenso del sentimiento religioso a lo largo de la curva (por tanto, la correlación negativa entre ingreso y religiosidad se debe al efecto sustitución), pero un aumento del ingreso y más altos niveles de religiosidad pueden ocasionar el desplazamiento hacia arriba y hacia la derecha de la curva de indiferencia. Obviamente todo este razonamiento acepta el supuesto de partida de que la religiosidad es una mercancía adquirible en un marco de libertad de elección del consumidor racional.

Finalmente, Hojman \& Miranda (2018) retoman la noción de la libertad como agencia (capacidad de control) y dignidad, en definitiva, el tercer concepto de libertad, y explorar su conexión con el bienestar en el contexto de una sociedad muy desigual y racista como es Chile. La hipótesis es que la agencia está relacionada positivamente con el bienestar subjetivo y que aquellos individuos que experimentan vergüenza o discriminación porque no se respeta su dignidad $-\mathrm{y}$ aquí se pueden recuperar los planteamientos de Honneth y Butler- disfrutan de menores niveles de bienestar subjetivo, si bien, después de controlar este último parámetro por la personalidad, se convierte en estadísticamente insignificante, lo que avala la existencia de preferencias o expectativas adaptativas. La explicación estaría en que dado que las personas que tienden a desarrollar preferencias adaptativas (como adaptación causal inconsciente de los deseos) son menos autónomas que las que operan bajo lógicas de planificación del carácter (como adaptación intencional consciente de los deseos), las preferencias adaptativas operarían con la lógica de la conformidad que explica la teoría religiosa de la privación (Stoltz, 2008). 
IV. Libertad de elegir como libertad económica del consumidor

El debate sobre los tres conceptos de la libertad, así como de los aportes de Sen y los estudios del desarrollo sobre la relación entre libertad y bienestar subjetivo o felicidad, muestran a las claras que este campo está respectivamente minado, contaminado y dominado por la doxa de la concepción negativa de la libertad de elección (aunque ampliada al final a la variante de libertad como no dominación) con tal carga de individualismo ético que se olvida la posibilidad de estudiar el "aspecto de los procesos" de la acción colectiva más allá de la mera invocación retórica. En este punto los críticos de Sen tienden a la unanimidad (Boltvinik, 2008a; 2008b; Crocker y Robeyns, 2010; Pettit, 2010; Ontano, 2015).

Para escapar de esa jaula teórica (que es también ontológica, epistemológica y, por encima de todo, axiológica) es necesario cuestionarse de nuevo el concepto de libertad de elección como sentido común (y significante vacío) reducible a libertad económica del consumidor. Desde que Milton y Rose Friedman publicaron en 1962 Capitalismo y libertad y luego recogieron los comentarios del Nobel en un popular programa de televisión de EEUU en un libro titulado Libertad de elegir (1980), este lema ha pasado a la cultura política occidental. Y lo ha hecho dentro del framming neoliberal de Friedman por medio de un silogismo hipotético de dos premisas (falsadas empíricamente) y una conclusión (verdadera solo lógicamente). La premisa 1 es que el mercado (gobierno) favorece (perjudica) "la libertad en los acuerdos económicos"; la premisa 2 expresa que la libertad económica es "un medio indispensable para el logro la libertad política" (Friedman \& Friedman 1962, p.8). Siguiendo el modus ponens característico del neopositivismo de Friedman (que viola el canon popperiano), la conclusión lógica (pero falaz) se puede formular de la siguiente manera: la libertad negativa de elegir es la esencia de la "libertad ampliamente entendida" (Friedman \& Friedman, 1962, p.8), con lo que "la libertad humana y la libertad económica corren juntas" (Friedman \& Friedman, 1980, p.309).

Pettit (2016, p.17) critica este concepto neoliberal (y escolástico) de libertad de elegir porque parte del supuesto ficticio de que "nadie pierde libertad en virtud de los acuerdos económicos consentidos, sin importar cuán débil y explotable sea la posición de negociación que ocupa". Pero su solución-típicamente liberal, compartida por Mill y Sen-de aumentar el poder compensatorio de la parte más débil empoderándola con derechos, deja intacta la distribución prevalente de la riqueza y del ingreso, que es justamente lo que se necesitaría alterar mediante acción colectiva para que la libertad como no dominación fuera de todos (en forma de capacidades colectivas) y no solo de unos pocos como ya reclamó Polanyi. Ello remite a la crítica que hace Boltvinik (2008b, p.30) de Sen: "la verdadera libertad... se alcanza, como nos ayudan a comprender Fromm y Maslow, cuando la necesidad no obliga", lo que significa volver al concepto de libertad positiva de Marx, que se puede considerar como verdadero punto de partida del desarrollo como ampliación de las capacidades o desarrollo humano (Lebowitz, 2010; Düzenli, 2016), como en su día reconoció el propio Sen (1983). .9 Marx (1894, p.807) afirma que "el reino de la libertad solo empieza allí donde termina el trabajo impuesto por la necesidad y por la

\footnotetext{
9 En una entrevista posterior y a propósito del bienestar humano, Sen defiende "dar a cada individuo el máximo de libertad para dirigir su vida como quiera. Como escribe Marx, hay que sustituir el dominio de las circunstancias sobre la vida humana por el dominio de los seres humanos sobre las circunstancias. Dar a los seres humanos la libertad: tal es el fin que tiene, a mi modo de ver, su definición más clásica en los Manuscritos económico-filosóficos de 1844... El Marx de 1844 expresó, probablemente con más fuerza que cualquier otro pensador, la idea de progreso en términos de libertad humana" (Sen, 1993, p.78)
} 
coacción de los fines externos". Por tanto, es necesario recuperar su denuncia de la ideología liberal de la no interferencia en el contexto de la crítica al capital como relación social entre mercancías: el "Edén de los derechos humanos innatos" en el que imperan "la libertad, la igualdad, la propiedad y Bentham" (Marx, 1867, p.186). Si de la esfera de la producción (el contrato de trabajo asalariado que expresa la relación social del capital) se pasa a la de la circulación (al mercado de bienes y servicios donde ser revela la libertad de elección de los consumidores a través de las preferencias de compra), es necesario también tener presente la crítica del capitalismo cultural realizada por los teóricos de la Escuela de Frankfurt y en concreto por Herbert Marcuse cuando desmenuza la libertad del consumidor y su paradójica "conciencia feliz". ${ }^{10}$ La novedad del planteamiento de Marcuse es que analiza esta operación de "la ideología represiva de la libertad" (Marcuse, 1964, p.43) a través del lenguaje. La misma operación ideológica se repite también, según Slavoj Žižek (2008, p.186) en la esfera política con la "elección forzada".

$Y$ en este capitalismo revolucionado de la felicidad paradójica, la civilización del hiperconsumo que resulta "el nuevo opio del pueblo" (Degli Esposti, 2012; Matusitz, 2012), el elevado bienestar material convive con una creciente insatisfacción psicológica. Este es el trasfondo que analizan-desde el ámbito de los estudios de la psicología social- Markus \& Schwartz (2010) en su abordaje de la relación entre la libertad de

\footnotetext{
${ }^{10}$ Tal paradoja consiste en que "las personas se identifican con la existencia que se les impone y encuentran en ella su propio desarrollo y satisfacción" (Marcuse 1964, p.13) sobre la base de la satisfacción de "necesidades falsas" creadas por la publicidad, y que impiden "el desarrollo de la capacidad" pero generan "una euforia dentro de la infelicidad" (Marcuse 1964, p.7). Para Marcuse (1964, pp.9-10), "la abrumadora necesidad de producir y consumir el despilfarro" se encuentra en que "el rango de elección abierto al individuo no es el factor decisivo en la determinación de la libertad humana, sino lo que puede ser elegido y lo que es elegido por el individuo".
}

elegir y el consumo. Los autores proceden a desmontar el silogismo friedmanita transitivo en su variante de los estudios sobre el bienestar: si A (mayor libertad y autonomía) implica B (mayor bienestar); y C (mayor rango de elección) luego $A$ (mayor libertad $y$ autonomía); entonces $C$ (mayor rango de elección) luego B (mayor bienestar). Según Markus \& Schwartz (2010), todo el proceso de socialización en EEUU y de los países de Europa occidental se basa en la reproducción de individuos independientes que sean capaces de realizar sus propias elecciones, de acuerdo a un esquema inside-out, donde el propósito de la vida es descubierto desde dentro de cada individuo y la noción de auto-expresión se considera como la suprema aspiración de este. Sin embargo, los principales experimentos realizados con personas de distintas culturas y procedencias (Este y Sur de Asia) y, dentro de EEUU, con diferentes clases sociales, muestra que elección, libertad, autonomía y bienestar no están inevitablemente relacionados: de hecho, el silogismo transitivo solo funciona para los individuos de la clase media de EEUU, mientras que los asiáticos no han recorrido el camino que va del modelo outisde-in (donde el individuo se define en términos de sus relaciones con otros individuos y se realiza a través de la participación en entidades más amplias) al inside-out. El repaso de la literatura de psicología cognitiva muestra que la elección es buena, pero que más posibilidad de elección no es mejor y que la elección por sí misma no es lo único bueno: la "sobrecarga de elección" puede conducir a la parálisis, decisiones subóptimas o la insatisfacción incluso con decisiones correctas de consumo, y, lo que es peor, puede ser responsable de la intensificación de patologías sociales como la depresión, la ansiedad y toda clase de desórdenes asociados a la proliferación de adicciones (Markus \& Schwartz, 2010, p.351). En resumen, la elección y la relación entre elección y liber- 
tad están socialmente construidas, dicha construcción -que se toma como dadapuede resultar debilitante en vez de liberadora y no sirve para calmar la búsqueda del sentido de la vida. La recomendación de los autores es "poner más atención a la «libertad para»-a las condiciones que permiten vivir las buenas vidas-, [lo que] podría tener como resultado que exista demasiada «libertad de»", de forma que se necesiten más restricciones de parte del Estado, la familia, la escuela o las instituciones culturales y religiosas (Markus \& Schwartz, 2010, p.352).

Recapitulando, lo que entienden los encuestados en la mayor parte de las sociedades por libertad de elegir forma parte de un sentido común compartido, en términos cognitivos y evaluativos, enmarcado en una determinada cultura política que, a su vez, experimentan inconscientemente como portadores y usuarios (Cartocci, 2011). Ello implica que las encuestas para medir el bienestar subjetivo podrían incurrir en los dos clásicos efectos de sesgo cognitivo: el "efecto de falso consenso" (Gilovich, 1991, pp.112-113) o "tendencia a permitir que las creencias de otros influyan en las nuestras", y el "efecto reflectante" que es "un rebote de lo que sostienen los medios de comunicación" (Sartori 1997, p.88). En todo caso, queda abierta una de las "cuestiones fundamentales que surgen cuando se considera el aspecto humano de la libertad" que planteó Fromm (1941, p.30): "¿se trata [la libertad] de una experiencia idéntica, cualquiera que sea el tipo de cultura a la cual una persona pertenece, o se trata de algo que varía de acuerdo con el grado de individualismo alcanzado en una sociedad dada?"

\section{Consideraciones finales}

Para responder a esa pregunta es necesario intentar una nueva operacionalización de la relación entre los dos conceptos de libertad partiendo del análisis que hace Marx del fetichismo de la mercancía, ${ }^{11}$ que conduce a cuestionar la tesis eurocéntrica, planteada en el apartado tres de este trabajo, de que la religiosidad y la libertad son componentes sustitutivos en el proceso de modernización.

Si la libertad de elección y los elementos de control que la acompañan funcionan para la gente a modo de creencias religiosas seculares, entonces no existiría el efecto sustitutivo entre religiosidad y libertad que apunta la teoría postmaterialista de la modernización, y dicho efecto solo sería válido para los países occidentales de Europa que, de ser el referente de la teoría de la secularización (como manifestación en el nivel de las mentalidades del proceso de modernización) se han acabado convirtiendo en la excepción con la teoría de la de-secularización ante el "retorno de la religión" y "su estruendoso reingreso en el campo político" (Keucheyan, 2016, p.50) a partir de la década de 1980 (Hjelm, 2012; Fokas, 2012).

Ello conduce a una ampliación conceptual que desborda el objetivo de esta revisión. Mientras tanto, cabe concluir que también justifica la recuperación que aquí se ha propuesto del concepto de libertad positiva en la tradición aristotélico-marxista-polanyiana y su entronque con el planteamiento original de Sen. Porque en ese estadio postmaterialista, en el cual las "formas flexibles de religión espiritual individual" tienden a resurgir (Inglehart \& Welzel, 2005, pp.3132; Klein, 2011) no hay nada nuevo bajo el sol teórico, como muestra la crítica premonitoria de Fromm a la reducción de la libertad a libertad de elección, reducción que convirtió el individualismo en una "cascara vacía" Fromm (1941, p.308). El razonamiento del Fromm no puede ser más actual porque para este autor "el futuro de la democracia

\footnotetext{
"Vale recordar que Marx describió la mercancía como "un objeto endemoniado, rico en sutilezas metafísicas y reticencias teológicas" (Marx 1867, p.81) y que al hablar del fetichismo de la mercancía dijo que era la auténtica "religión de la vida cotidiana" (Marx 1894, p.817).
} 
depende de la realización del individualismo", pero no en el sentido del individuo que ejerce su libertad negativa (individualismo posesivo), sino que disfruta de la libertad positiva (individualismo progresivo): "la victoria de la libertad es solamente posible si la democracia llega a constituir una sociedad en la que el individuo, su desarrollo y felicidad constituyan el fin y el propósito de la cultura" (Ibíd.).

\section{Referencias}

Berlin, I. ([1969] 2002). Five essays on liberty, en H. Hardy, ed., Liberty, incorportating Four Essays in Liberty, Oxford: Oxford University Press, pp. 1-282.

Berlin, I. ([1998] 2002). Final retrospect, en H. Hardy, ed., Liberty, incorportating Four Essays in Liberty, Oxford: Oxford University Press, pp. 322-330.

Berlin, I. ([2002] 2014). Freedom and Its Betrayal. Six Enemies of Human Liberty. Princenton: Princenton University Press.

Beytía, P. (2016). The Singularity of Latin American Patterns of Happiness, en M. Rojas, ed., Handbook of Happiness Research in Latin America. Dordrecht: Springer, pp. 17-30.

Bjerke, A.R. (2012). Modernization, en M. Juergensmeyer y W.C. Roof, eds., Encyclopedia of Clobal Religion. Los Angeles: SACE, vol. 2, pp. 810-813.

Boltvinik, J. (2008a). Evaluación crítica del enfoque de «capabilities» de Amartya Sen (Primera Parte), Mundo Siglo XXI, 12, pp. 43-55.

Boltvinik, J. (2008b). Evaluación crítica del enfoque de «capabilities» de Amartya Sen (Segunda Parte), Mundo Siglo XXI, 13, pp. 19-41.

Burns, T. (2016). «Happy slaves»? The adaptation problema and identity politics in the writings of Amartya Sen. International Journal of Social Economics, 43(12), pp. 1178-193.
Cano, M. (2017). Agencia crítica y desposesión. La actualidad de la pregunta por la libertad en Judith Butler. Isegoría. Revista de Filosofía Moraly Política, 56, pp. 263-277.

Cartocci, R. (2011). Political Culture, en B. Badie, D. Berg-Schlosser y L. Morlino, eds., International Encyclopedia of Political Science. Los Angeles: SAGE, vol. 6, pp. 1967-1979.

Casassas, D. (2008). En torno a las condiciones materiales de la libertad: la renta básica como fundamento de la sociedad civil. Revista Latinoamericana de Filosofía, 34(1), pp. 134-157.

Casassas, D. (2018). Para la constitución de la república contemporánea: entrevista a David Casassas, Recuperado de: http://www.sinpermiso.info/textos/para-la-constitucion-politica-de-la-republica-contemporanea-entrevista-a-david-casassas [consulta 20 de diciembre de 2018].

Croker, D.A. \& I. Robeyns (2010): Capability and Agency, en C.W. Morris, ed. , Amartya Sen, Nueva York: Cambridge University Press, pp. 60-90.

Dang, A.T. (2014). Amartya Sen's Capability Approach: A Framework from Well-Being Evaluation and Policy Analysis? Review of Social Economy, 72(4), pp. 460-484.

Degli Esposti, P. (2012). Hyperconsumption, en G. Ritzer, ed., The Wiley-Blackwell Encyclopedia of Globalization, Oxford: Blackwell Publishing.

Dobbelaere, K. (2007). Secularization, en G. Ritzer, ed., The Blackwell Encyclopedia of Sociology. Oxford: Blackwell Publishing, pp. 4148-4156.

Düzenli, F.E. (2016). Surplus-producing labour as capability: a Marxian contribution to Amartya Sen's revival of political economy. Cambridge Journal of Economics, 40(4), pp. 1019-1035.

Fokas, E. (2012): Secularization, en M. Juergensmeyer y W.C. Roof, eds., Encyclopedia ofClobal Religion. Los Angeles: SAGE, vol. 2, pp. 1143-1145. 
Frey, B.S. \& A. Stulzer (2000). Happiness, Economy and Institutions. The Economic Journal, 110, pp. 918-938.

Friedman, M. \& R. Friedman ([1962] 2002). Capitalism and Freedom. 40th Anniversary Edition With a new Preface by the Author, Chicago: The University of Chicago Press.

Friedman, M. \& R. Friedman (1980). Free to Choose, Nueva York: HB].

Fromm, E. ([1941] 2005). El miedo a la libertad, Buenos Aires: Paidós.

Gilovich, T. (1991). How We Know What Isn't So. The Fallibility of Human Reason in Everyday Life, Nueva York: The Free Press.

Gundlach, E. \& M. Opfinger (2013). Religiosity as a Determinant of Hapiness. Review of Development Economics, 17(3), pp. 523-539.

Hayek, F.A. von ([1938] 1940). La libertad y el sistema económico. El Trimestre Económico, 6(24-4), pp. 666-700.

Hayek, F.A. von ([1944] 2001). The Road to Serfdom, Londres, Routledge.

Helliwell, J.F., H. Huang \& S. Wang (2017). The Social Foundations of World Hapiness, en ].F. Heliwell, R. Layard \& ]. Sachs, eds., World Happiness Report 2017, Nueva York, Sustainable Development Solutions Network, pp. 8-47.

Hjelm, T. (2012). Desecularization, en M. Juergensmeyer \& W.C. Roof, eds., Encyclopedia of Clobal Religion. Los Angeles, SAGE, vol. 1, pp. 293-294.

Hirai, T., F. Comin, y Y. Ikemoto (2016). Happiness and human development: $A$ capability perspective. Journal of Human Development, 28(7), pp. 1155-1169.

Hojman, D.A. \& A. Miranda (2018). Agency, Human Dignity, and Subjective Well-being. World Development, 101, pp. 1-15.

Honneth, A. (2006). El reconocimiento como ideología. Isegoría. Revista de Filosofía Moraly Política, 35, pp. 129-150.

Horkheimer, M. ([1962] 1986). Sobre el concepto de libertad, en M. Horkheimer, Sociedades en transición: estudios de filosofía social. Barcelona: Planeta-DeAgostini, pp. 11-20.
Inglehart, R. \& C. Welzel (2005). Modernization, Cultural Change, and Democracy, Nueva York, Cambridge University Press. Inglehart, R. \& C. Welzel (2007). Modernization, en G. Ritzer, ed. The Blackwell Encyclopedia of Sociology. Oxford, Blackwell Publishing, pp. 3071-3078.

Inglehart, R. et al. (2008). Development, Freedom, and Rising Happiness. A Global Perspective (1981-2007). Perspectives on Psychological Science, 3(4), pp. 264-285.

Keucheyan, R. (2016). Las mutaciones de la teoría crítica. Un mapa del pensamiento radical hoy. Nueva Sociedad, 261, pp. 37-53.

Kingdon, G.G. \& ]. Knight (2006). Subjective Well-Being Poverty vs. Income Poverty and Capabilities Poverty? Journal of Development Studies, 42(7), pp. 1199-1224.

Klein, M. (2011). Postmaterialism, en B. Badie, D. Berg-Schlosser \& L. Morlino, eds., International Encyclopedia of Political Science, Los Angeles, SAGE, vol. 6, pp. 20932095.

Knutsen, O. (2011): Values, en B. Badie, D. Berg-Schlosser \& L. Morlino, eds., International Encyclopedia of Political Science, Los Angeles, SACE, vol. 8, pp. 2691-2697.

Kramer, M.H. (2011). Liberty, en B. Badie, D. Berg-Schlosser \& L. Morlino, eds., International Encyclopedia of Political Science, Los Angeles, SACE, vol. 5, pp. 1447-1458.

Kukathas, C. (2007). Liberty, en R.E. Goodin, P. Pettit \& T. Pogge, eds., A Companion to Contemporary Political Philosophy, Oxford, Blackwell Publishing, vol. 2, pp. 685-698.

Lawson, T. (1997). Economics and reality: Economics as social theory. Londres y Nueva York, Routledge.

Lebowitz, M.A. (2010). The Socialist Alternative. Real Human Development. Nueva York, Monthly Review Press.

Marcuse, H. ([1964] 2002). One-Dimensional Man. Studies in the ideology of advanced industrial society. Nueva York, Routledge.

Markus, H.R. \& B. Schwartz (2010). Does Choice Mean Freedom and Well-Being?, Journal of Consumer Research, 37(2), pp. 344-355. 
Marx, K. ([1867] 1996). Capital. A Critique of Political Economy. Volume I, en K. Marx \& F. Engels, Collected Works, Nueva York, Progress Publishers, vol. 35.

Marx, K. ([1894] 1998). Capital. A Critique of Political Economy. Volume III, en K. Marx \& F. Engels, Collected Works. Nueva York, Progress Publishers, vol. 37.

Matusitz, J. (2012). Cathedrals of consumption, en G. Ritzer, ed., The Wiley-BlackweII Encyclopedia of Globalization, Oxford, Blackwell Publishing.

Müller, T., N.D. De Craaf, \& P. Schmidt. (2014). Which Societies Provide a Strong Religious Socialization Context? Explanations Beyond the Effects of National Religiosity. Journal for the Scientific Study of Religion, 53(4), pp. 739-760.

Norris, P. \& R. Inglehart (2011). Sacred and Secular. Religion and Politics Worldwide. Nueva York, Cambridge University Pres.

Otano, G. (2015). La libertad como relación social: una interpretación sociológica del enfoque de las capacidades de Amartya Sen. Revista Iberoamericana de Estudios del Desarrollo, 4(1), pp. 98-127.

Pereira, G. (2008). Preferencias adaptativas: un desafío para el diseño de las políticas sociales, Isegoría. Revista de Filosofía Moral y Política, 36, pp. 143-165.

Pettit, P. (1997). Republicanism. A Theory of Freedom and Government. Oxford, Oxford University Press.

Pettit, P. (2009). De la República a la Democracia. Revista Internacional de Pensamiento Político, 4, pp. 47-68.

Pettit, P. (2010). Feedom in the Spirit of Sen, en C.W. Morris, ed., Amartya Sen. Nueva York, Cambridge University Press, pp. 91114.

Pettit, P. (2012). On the People's Terms. A Republican Theory and Model of Democracy, Cambridge, Cambridge University Press.

Pettit, P. (2016). A Brief History of Liberty And Its Lessons. Journal of Human Development and Capabilities, 17(1), pp. 5-21.
Polanyi, K. ([1944] 2001). The Great Transformation. Political and Economic Origins of Our Time, Boston, Beacon Press.

Rojas, M. (2016). Happiness, Research, and Latin America, en M. Rojas, ed., Handbook of Happiness Research in Latin America, Dordrecht, Springer, pp. 1-13.

Ryan, R.M. \& E.L. Deci. (2000). Self-Determination Theory and the Facilitation fo Intrinsic Motivation, Social Development, and Well-Being. American Psycologist, 55(1), 68-78.

Sartori, G. ([1997] 2005). Homo videns. La sociedad teledirigida. Madrid: Punto de Lectura.

Sen, A.K. (1983). Development: Which Way Now. The Economic Journal, 372, pp.745762.

Sen, A.K. (1985). Agency and Freedom: The Dewey Letures 1984. The Journal of Philosophy, 82(4), pp. 169-221.

Sen, A.K. (1993). La libertad positiva. Entrevista con Amartya Kumar Sen, Debats, 43/44, pp. 77-83.

Sen, A.K. (1999). Development as Freedom. Nueva York, Alfred A. Knopf.

Sen, A.K. (2009). The Idea of Justice, Cambridge, Harvard University Press.

Skinner, Q. (2002). A Third Concept of Liberty. Isaiah Berlin Lecture. Proceedings of British Academy, 117, pp. 237-268.

Steinmetz, G. (1998). Critical Realism and Historical Sociology. A Review Article, Comparative Studies in Society and History, 40(1), pp. 170-186.

Stewart, F. (2014). Against Happiness: A Critical Appraisal of the use of measures of Happiness or evaluation progress in development, Journal of Human Development and Capabilities, 15(4), pp. 239-307.

Stoltz, ]. (2008). Explaining religiosity: towards a unified theoretical model, The British Journal of Sociology, 60(2), pp. 345376.

Verme, P. (2009). Happiness, freedom and control, Journal of Economic Behavior \& Organization, 71(2), pp. 146-161. 
Volpe, T. (2010). Modernization Theory, en M. Bevir, ed., Encyclopedia of Political Thought, Londres, Sage, pp. 895-899.

Welzel, C. (2011). "Human Development", en B. Badie, D. Berg-Schlosser \& L. Morlino, eds., International Encyclopedia of Political Science, Los Angeles, SAGE, vol. 4 , pp. 1101-1104.

Žižek, S. (2008). The Sublime Object of Ideology, Nueva York: Verso.

\section{Nota}

*Este trabajo forma parte del proyecto de la Universidad de Cantabria "Bienestar subjetivo y desarrollo humano: explicando la paradoja latinoamericana” UC2016-GRE-05. Agradezco a Borja López Noval sus sugerencias, comentarios y revisión del texto. Los errores y omisiones son de mi exclusiva responsabilidad. Salvo indicación contraria, todas las cursivas de las citas están en el original. 